Ann. Zootech., I973, $22(3), 3 \mathrm{II}-326$.

\title{
INFLUENCE DE L'APPORT ÉNERGÉTIQUE SUR L'UTILISATION DIGESTIVE ET MÉTABOLIQUE DES NUTRIMENTS, ET LES PERFORMANCES DE REPRODUCTION, CHEZ LA TRUIE GESTANTE NULLIPARE
}

\author{
M. ÉTIENNE et Y. HENRY \\ avec la collaboration technique de J. Lebost, F. Cointepas, \\ Christiane Vachot, Chantal Potier et Michèle Seguin \\ Station de Recherches sur l'Élevage des Porcs, \\ Centre national de Recherches zootechniques, I. N.R. A., \\ 78350 Jouy en Josas
}

\begin{abstract}
RÉSUMÉ
Une expérience a été réalisée sur des truies gestantes nullipares, de race Large White, saillies à un poids voisin de $120 \mathrm{~kg}$, dans le but d'étudier l'influence du niveau de l'apport quotidien d'énergie sur les coefficients d'utilisation digestive des principes énergétiques et azotés, la rétention azotée et la synthèse des lipides chez la Truie, ainsi que sur la composition chimique des porcelets à la naissance. A cet effet, 3 lots de 3 animaux sont mis en comparaison, qui reçoivent respectivement une ration témoin, à base de maïs et de tourteau de soja (lot I), la même additionnée de rafles (lot 2) ou d'amidon de maïs (lot 3 ).

L'élévation du taux de cellulose dans le régime des truies du lot 2 provoque un abaissement de tous les coefficients de digestibilité mesurés. Les teneurs en énergie digestible des rafles et de l'amidon de mais, rapportées à la matière sèche, sont respectivement de I 900 et $4200 \mathrm{kcal} / \mathrm{kg}$. La quantité d'azote retenu chaque jour par les truies ne varie pas avec l'avancement de la gestation, mais augmente avec le niveau d'énergie digestible. Cette amélioration est importante surtout pour les animaux du $2^{\text {e }}$ lot par rapport à ceux du lot témoin. La taille et lo poids des portées, de même que la composition chimique des porcelets à la naissance, ne sont pas affectés par l'alimentation maternelle. Seul, le gain net de gestation des truies est significativement augmenté par l'élévation du niveau énergétique des rations.

Sur le plan pratique, ce travail montre qu'un rationnement exagéré en énergie digestible nuit aux possibilités de rétention azotée des truies gestantes nullipares, et que les recommandations actuelles (N. R. C., I968) sont marginales pour des animaux n'ayant pas encore terminé leur croissance.
\end{abstract}




\section{INTRODUCTION}

La mise en évidence du phénomène d'anabolisme gravidique chez la Truie, notamment par SALMON-L,EGAGNEUR et JACQUOT (I96I), LODGE et al. (I96I), a permis de préciser les résultats antérieurs d'Evans (I929) et MíTcheis et al. (I93I), selon lesquels une grande partie des nutriments ingérés par l'animal gestant est utilisée à son profit pour la synthèse de tissus maternels extra-utérins. Cette synthèse, qui est effectuée avec un rendement supérieur à celui de témoins non gestants, suppose une diminution du besoin énergétique d'entretien, ainsi qu'une meilleure utilisation de l'azote, liées à l'état de gestation.

Par ailleurs, la combinaison des mesures de bilans et des analyses corporelles (LENKEIT et al., I955 ; MoUSTGAARD, I962) indique que les besoins propres au développement des embryons et de letrs annexes sont faibles. Ceci explique que la distribution de rations modiques aux truies gestantes affecte peu les produits de la conception, ainsi que le démontrent de nombreux travaux (LODGE et al., Ig66; ELSLEY et al., I969; BAKER et al., I969). Seul le gain corporel de la mère est réduit.

Ainsi s'est trouvée démontrée la priorité de la fonction de reproduction sur celle de la croissance propre de la mère. Ces résultats ont conduit à proposer une diminution importante des normes alimentaires pour les truies gestantes. Par exemple, dans le cadre du National Research Council, elles sont passées de 9800 kcal d'énergie digestible/j en I964 à 6600 en I 968 pour des truies adultes. Cependant, il existe encore actuellement des divergences dans les recommandations suivant les différents pays. Elles s'expliquent en partie par la variabilité élevée des critères zootechniques qui ont servi à établir ces normes d'alimentation.

Alors que le besoin azoté de gestation a fait l'objet de nombreuses estimations par la méthode des bilans (Mrlier et al., I969; PIkE, I970; RipPEi, I965 ; SALmoNLEGAGNEUR, I965), peu de travaux du même type ont été effectués en ce qui concerne l'énergie. Il semble, d'après les résultats de SALMON-LEGAGNEUR (I963), que le niveau énergétique de la ration constitue davantage un facteur limitant de l'anabolisme protidique que le niveau azoté lui-même, tout au moins par rapport aux recommandations en vigueur. D'autre part, l'influence de la nature des nutriments énergétiques a été peu étudiée. La comparaison de l'apport d'une partie de l'énergie sous forme d'amidon de céréales ou de lipides (SALMON-I,EGAGNEUR, I969) indique toutefois que la rétention azotée serait plus favorisée par l'élévation des apports quotidiens d'énergie digestible que par la présence de graisses per se dans le régime.

L'expérience que nous rapportons ici a eu pour but d'étudier, chez la Truie nullipare gestante, l'influence du niveau de l'apport d'énergie digestible et du volume de la ration sur la gestation. Pour ce faire, une ration de base considérée comme témoin a été supplémentée, soit par des glucides hautement digestibles (amidon de maïs), soit par un aliment cellulosique (rafles de maïs). La comparaison de ces régimes a porté sur l'utilisation digestive des principes alimentaires, la rétention azotée, les variations quantitatives et qualitatives des dépôts gras chez la Truie au cours de différentes phases de la gestation. Par ailleurs, l'influence éventuelle du rationnement 


\section{TABLEAU I}

Composition des régimes expérimentaux (p. Iоo)

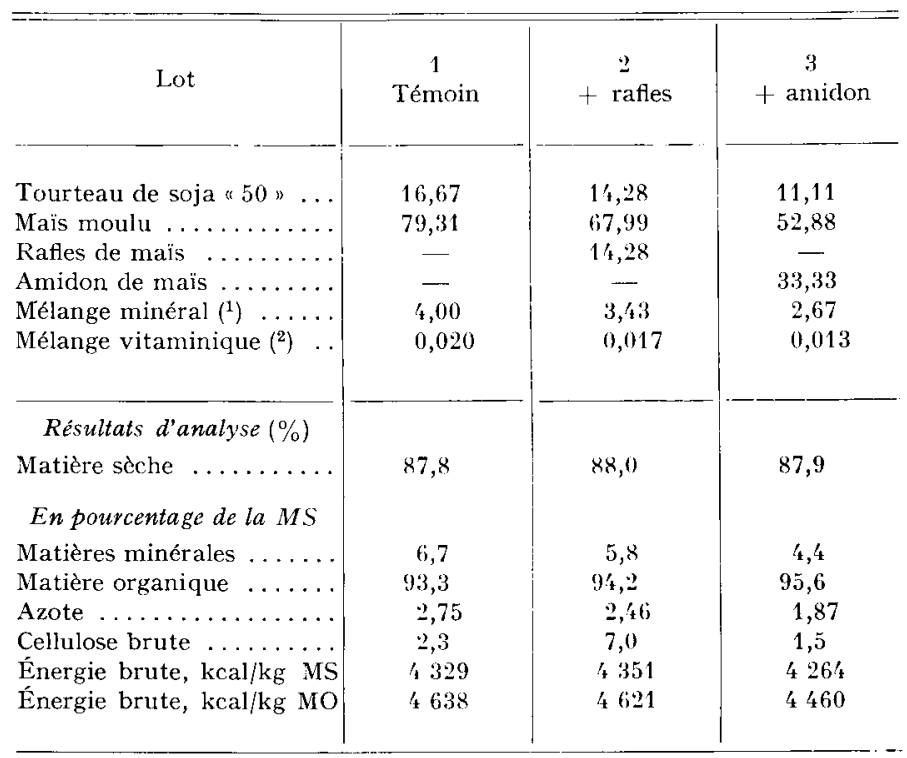

(1) Composition, p. 100 du mélange minéral :

Phosphate bicalcique ......... 40

Carbonate de calcium ......... 22

Chlorure de sodium .......... 25

Carbonate de magnésium ........ 10

Sulfate ferreux ............ 1

Sulfate de zinc ............ 1

Sulfate de manganèse $\ldots \ldots \ldots \ldots \ldots, 0,1$

Sulfate de cuivre ........... 0,05

Sulfate de cobalt ........... 0,05

Iodure de potassium stabilisé $\ldots \ldots . \quad 0,01$

(2) Composition du mélange vitaminique :

Vitamine $\mathrm{D}_{3}(100000 \mathrm{UI} / \mathrm{g}) \quad \ldots \ldots \ldots \quad 0,25$

Riboflavine $\ldots \ldots \ldots \ldots \ldots \ldots \ldots \ldots . \quad 0,10$

Acide ascorbique $\ldots \ldots \ldots \ldots \ldots \ldots \ldots, \quad 0,10$

Nicotinamide .............. 0,20

Pantothénate de calcium ....... 0,25

Vitamine A $(50000 \mathrm{UI} / \mathrm{g}) \ldots \ldots \ldots . \quad 2,0$

Vitamine $B_{12}(100 \mathrm{mg} / \mathrm{kg}) \ldots \ldots \ldots \quad 2,0$

Choline (25 p. 100) . ......... 20,0

Méthionine ............. 10,0

Glucose anhydre q.s.p. $100 \mathrm{~g}$..... 
des mères gestantes sur les fœetus a été recherchée par l'étude de la composition chimique des porcelets à la naissance. Enfin, les performances zootechniques ont, dans une moindre mesure, complété ces résultats.

\section{MATÉRIEI, ET MÉTHODES}

Neuf truies nullipares, de race Large White, sont saillies au premier cestrus après qu'elles aient atteint le poids de I $20 \mathrm{~kg}$. Trois lots de 3 animaux chacun sont alors constitués, différant par le niveau et la nature des nutriments énergétiques, mais recevant un apport quotidien égalisé de protéines. Les truies du lot témoin (lot $\mathrm{I}$ ) ne consomment que $\mathrm{I}, 8 \mathrm{~kg}$ d'une ration de base composée de maïs et de tourteau de soja, tandis que celles des autres lots disposent en plus de $0,3 \mathrm{~kg}$ de rafles de maïs à $89,7 \mathrm{p}$. I oo de matière sèche (lot 2) ou de $0,9 \mathrm{~kg}$ d'amidon de maïs à 88, I p. Ioo de matière sèche (lot 3). Compte tenu de la faible teneur en azote des rafles de mais $(0,55 \mathrm{~g} / \mathrm{kg})$, tous les animaux ingèrent quotidiennement la même quantité de protéines de qualité identique; il en va de même pour les minéraux et les vitamines. Les rations sont distribuées en 2 repas égaux par jour. Le mélange des matières premières qui entrent dans la composition des différents aliments est effectué lors de chaque fabrication. Le tableau I rapporte la composition des régimes. Les truies restent pendant un mois au sol, a fin de s'assurer qu'elles sont bien gestantes. Celles qui reviennent en chaleurs sont éliminées de l'expérience, et remplacées par d'autres. Les animaux sont ensuite soumis à 4 périodes de collecte de 4 jours, en cages de digestibilité, pendant lesquelles on récolte séparément les fèces et l'urine, selon une technique décrite par ailleurs (Salmon-Legagneur, 1965). Chaque période de collecte est précédée d'une semaine d'adaptation à la cage, et suivie d'une semaine au sol, en loges collectives avec alimentation individuelle. Les excréta sont collectés à partir des $38^{\mathrm{e}}, 59^{\mathrm{e}}$, $80^{\mathrm{e}}$ et $\mathrm{Io} \mathrm{I}^{\mathrm{e}}$ jours de gestation.

Sur les échantillons représentatifs moyens de chacune des fabrications d'aliments et les fèces, on procède aux déterminations suivantes : matière sèche par séchage à l'étuve à $95^{\circ} \mathrm{C}$, cendres par incinération au four à $50^{\circ} \mathrm{C}$, azote par la méthode Kjeldahl, énergie par combustion dans un calorimètre balistique "Féry ", cellulose brute par la méthode de Weende (modifiée O. C. D. E.).

Afin de suivre les variations de composition en acides gras du tissu adipeux et l'intensité de la synthèse des lipides in vitro, des fragments de 4 à $5 \mathrm{~g}$ de lard sont prélevés au niveau du cou des truies, avant le premier passage en cage de digestibilité, et avant la mise bas (soit en moyenne à 26 et 108 jours de gestation). Ils sont obtenus après anesthésie générale au thiopental sodique, les animaux ayant été maintenus à jeun depuis la veille. Des coupes de ce tissu sont aussitôt incubées pendant 2 heures à $37^{\circ} \mathrm{C}$, en présence de glucose uniformément marqué au ${ }^{14} \mathrm{C}$, selon la technique mise au point par O'HeA et LEVEILle (r968), modifiée par HENRY (non publié). La mesure de la radioactivité du gaz carbonique produit, et des lipides synthétisés, fournit une évaluation de l'intensité de la lipogenèse. Le dosage des acides gras est effectué sur les mêmes fragments de lard par chromatographie en phase gazeuse (colonne de Chromosorb W. 20 p. Ioo DEGS sur Aerograph I 200 à ionisation de flamme), après saponification et méthylation des lipides extraits.

Les observations sur truies sont complétées par une étude de la composition chimique des porcelets à la naissance. Pour cela, dans chaque portée, un porcelet est sacrifié par asphyxie et conservé à $-25^{\circ} \mathrm{C}$. La composition chimique est déterminée après broyage et homogénéisation de l'organisme entier congelé, et lyophilisation d'un échantillon représentatif. Les teneurs en matière sèche, minéraux, azote et énergie sont estimées par les techniques déjà décrites, tandis que les lipides sont dosés par la méthode cle BLIGH et Dyer (I959).

Les données expérimentales sont traitées par l'analyse de variance, de manière à dissocier l'effet propre du régime de celui du stade de gestation.

\section{RÉSULTATS}

\section{A. - Utilisation digestive des nutriments énergétiques (tabl. 2)}

Ainsi que le prévoyait le protocole expérimental, l'ingestion azotée a été la même dans les 3 lots, de l'ordre de $44 \mathrm{~g} /$ jour. Celles de matière sèche, de matière organique et d'énergie brute varient de façon parallèle à la quantité d'aliment frais. Par contre, 
l'introduction de $300 \mathrm{~g}$ de rafles de maïs dans le régime des truies du lot 2 provoque une élévation du taux de cellulose brute de 2,3 à $7 \mathrm{p}$. Ioo et de son ingestion quotidienne, qui augmente de plus de 200 p. Ioo.

Les coefficients de digestibilité obtenus par le calcul de l'excrétion fécale indiquent tous un effet significatif $(\mathrm{P} \leqslant 0,00 \mathrm{I})$ du régime : les résultats du lot 3 sont, en ce qui concerne la matière sèche, la matière organique et l'énergie, supérieurs de 4 à 5 points à ceux du lot témoin, tandis que ceux du lot 2 sont inférieurs de 6 à 7 points. L'utilisation digestive de la cellulose brute est significativement plus faible dans le cas du régime qui en contient le plus (lot 2), soit 32 p. Ioo contre 46 et 53 respectivement dans les lots $I$ et 3 . Il faut toutefois remarquer que, d'une façon générale, la variabilité de ce critère est plus élevée que celle des autres, et plus particulièrement dans le cas $\mathrm{du} \operatorname{lot} 2$, pour lequel le coefficient de variation est de 47 p. Ioo.

\section{TABLEAU 2}

Utilisation digestive des nutriments énergétiques

\begin{tabular}{|c|c|c|c|}
\hline Lot & $\begin{array}{c}1 \\
\text { Témoin }\end{array}$ & $\begin{array}{c}2 \\
+ \text { rafles }\end{array}$ & $\begin{array}{c}3 \\
+ \text { amidon }\end{array}$ \\
\hline \multicolumn{4}{|c|}{ Nutriments ingérés/animal/j (g) } \\
\hline $\begin{array}{l}\text { Aliment frais } \ldots \ldots \ldots \ldots \ldots \\
\text { Matière sèche } \ldots \ldots \ldots \ldots \ldots \\
\text { Matière organique } \ldots \ldots \ldots \ldots \\
\text { Cellulose brute } \ldots \ldots \ldots \ldots \ldots \\
\text { Azote } \ldots \ldots \ldots \ldots \\
\text { Énergie brute }(\text { kcal } \ldots \ldots \ldots \ldots\end{array}$ & $\begin{array}{l}1800 \\
1580 \\
1475 \\
\\
\quad 37,6 \\
43,5 \\
6792\end{array}$ & $\begin{array}{c}2100 \\
1849 \\
1742 \\
128,2 \\
\quad 44,8 \\
7970\end{array}$ & $\begin{array}{c}2700 \\
2373 \\
2266 \\
37,5 \\
43,9 \\
10064\end{array}$ \\
\hline \multicolumn{4}{|c|}{ Coefficients de digestibilité $(\%)\left({ }^{1}\right)$} \\
\hline $\begin{array}{l}\text { CUD MS } \ldots \ldots \ldots \ldots \ldots \ldots \\
\text { CUD MO } \ldots \ldots \ldots \ldots \ldots \ldots \\
\text { CUD Énergie } \ldots \ldots \ldots \ldots \ldots \\
\text { CUD cellulose brute } \ldots \ldots \ldots \\
\text { CUD E E } \text { EUSC } \ldots \ldots \ldots \ldots \ldots \ldots\end{array}$ & $\begin{array}{l}85,8 \pm 0,5^{a} \\
89,0 \pm 0,5^{a} \\
86,5 \pm 0,5^{a} \\
46,1 \pm 2,0^{a} \\
87,1 \pm 0,6^{a}\end{array}$ & $\begin{array}{l}80,2 \pm 1,0^{b} \\
82,1 \pm 1,0^{b} \\
80,2 \pm 0,8^{b} \\
31,9 \pm 4,1^{b} \\
83,6 \pm 0,7^{b}\end{array}$ & $\begin{array}{l}91,1 \pm 0,5^{c} \\
93,4 \pm 0,4^{c} \\
91,1 \pm 0,4^{c} \\
53,5 \pm 2,9^{a} \\
92,1 \pm 0,5^{c}\end{array}$ \\
\hline \multicolumn{4}{|c|}{$\begin{array}{c}\text { Energie digestible des aliments } \\
(\mathrm{kcal})\left({ }^{1}\right)\end{array}$} \\
\hline $\begin{array}{l}\text { Énergie digestible, } \mathrm{kcal} / \mathrm{kg} \text { MS } \\
\text { Énergie digestible, } \mathrm{kcal} / \mathrm{kg} \mathrm{MO} \\
\text { Énergie digest.,kcal/animal/j.. }\end{array}$ & $\begin{array}{l}3716 \pm 24^{a} \\
3982 \pm 26^{a} \\
5873\end{array}$ & $\begin{array}{l}3455 \pm 34^{b} \\
3667 \pm 36^{b} \\
6388\end{array}$ & $\begin{array}{l}3877 \pm 32^{c} \\
4060 \pm 32^{a} \\
9201\end{array}$ \\
\hline
\end{tabular}

(1) Seuils de signification : * $: \mathrm{P} \leqslant 0,05 ; * *: \mathrm{P} \leqslant 0,01 ; * * *: \mathrm{P} \leqslant 0,001 ; \mathrm{NS}$ : Effet non significatif. Des lettres distinctes sur une même ligne indiquent que les valeurs auxquelles elles se rapportent sont statistiquement différentes.

Chaque valeur moyenne est accompagnée de son écart-type (12 données par lot).

Ces indications sont valables pour les tableaux suivants. 
Le coefficient d'utilisation digestive de l'énergie de la fraction non cellulosique de la ration (CUD $\mathrm{E}_{\mathrm{RSC}}$ ) (1) a été calculé selon des modalités décrites par ailleurs. (HENRY et ÉTIENNE, I 969), afin d'étudier l'effet propre de l'incorporation de cellulose sur l'utilisation de cette fraction. Les résultats vont dans le même sens que ceux de la digestibilité de la totalité de l'énergie, mais ils leur sont supérieurs, surtout pour les animaux du $2^{\mathrm{e}}$ lot. Ainsi, l'introduction de rafles de maîs dans le régime 2 provoque une diminution du coefficient d'utilisation digestive de la fraction non cellulosique de la ration, de l'ordre de 3 points (de 87 dans le lot I à 84 dans le lot 2).

Le stade de gestation n'exerce aucune influence sur les résultats précédents; de plus, il ne fait apparaître aucune interaction avec le régime. La teneur en énergie digestible de la matière sèche des aliments diffère entre les 3 lots; elle passe de $3877 \mathrm{kcal} / \mathrm{kg}$ pour le régime du lot 3 , à $3455 \mathrm{kcal}$ pour celui du lot 2 . Mais lorsque l'énergie digestible est rapportée à la matière organique, on n'observe plus d'écart entre les aliments contenant le moins de cellulose (4 o0o $\mathrm{kcal} / \mathrm{kg})$, au contraire du régime qui renferme des rafles de maïs (3667 $\mathrm{kcal} / \mathrm{kg}$ ).

\section{B. - Utilisation digestive et rétention des matières azotées (tabl. 3)}

La digestibilité des matières azotées du régime 2 est plus faible que celle des régimes I et 3 (83 p. Ioo contre 85 et 86 ), en raison d'un taux de cellulose plus élevé. Pour une même ingestion azotée, la quantité d'azote fixée quotidiennement augmente de I2,I à I5,o et I6,9 g respectivement dans les lots I, 2 et 3 ; il en est de même pour le coefficient de rétention azotée (CRN). Le coefficient d'utilisation pratique de l'azote (CUPN), résultant de la combinaison des critères précédents, est donc significativement abaissé chez les truies du lot témoin en comparaison de celles qui reçoivent de l'amidon de maïs. Les résultats des animaux du lot disposant de rafles sont intermédiaires.

TABLEAU 3

Digestibilité et rétention azotées

\begin{tabular}{|c|c|c|c|c|}
\hline Lot & $\begin{array}{c}1 \\
\text { Témoin }\end{array}$ & $\begin{array}{c}2 \\
+\quad \text { rafles }\end{array}$ & $\begin{array}{c}3 \\
+ \text { amidon }\end{array}$ & $\begin{array}{l}\text { Effet du } \\
\text { traitement }\end{array}$ \\
\hline \multicolumn{5}{|l|}{ Coefficient $(\%)$} \\
\hline CUD $N \ldots \ldots \ldots$ & $84,9 \pm 0,7^{a}$ & $83,2 \pm 0,9^{b}$ & $86,5 \pm 0,5^{a}$ & ** \\
\hline Azote retenu $(g / j) \ldots$ & $12,1 \pm 0,7^{a}$ & $15,0 \pm 0,9^{b}$ & $16,9 \pm 0,9^{b}$ & $* * *$ \\
\hline CRN $\ldots \ldots \ldots \ldots$ & $32,9 \pm 2,1^{a}$ & $40,0 \pm 2,4^{b}$ & $44,4 \pm 1,6^{b}$ & * \\
\hline CUP $N \ldots$ & $27,6 \pm 1,8^{a}$ & $33,2 \pm 2,0^{a, b}$ & $38,4 \pm 1,5^{b}$ & $* *$ \\
\hline
\end{tabular}

(1) $\mathrm{CUD} \mathrm{E}_{\mathrm{RSC}}=\frac{\text { Énergie totale absorbée - Énergie de la cellulose digérée }}{\text { Énergie totale ingérée - Énergie de la cellulose ingérée }}$, ounergie de la cellulose ingérée est obtenue en multipliant la quantité ingérée par la valeur énergétique brute de la cellulose $(4,127 \mathrm{kcal} / \mathrm{g}$ de matière sèche); ]'énergie de la cellulose digérée est égale à l'énergie de la cellulose ingérée multipliée par le CUD de la cellulose pour le régime considéré. 
Le stade de gestation n'a d'influence sur aucun de ces critères (tabl. 4). Il faut remarquer toutefois que la quantité d'azote fixé chaque jour par les truies du lot 3 tend à augmenter de $\mathrm{I} 5,6$ à $\mathrm{I} 8, \mathrm{I} \mathrm{g} /$ jour $(\mathrm{P} \leqslant 0, \mathrm{IO})$ entre $\mathrm{I}$ mois après la saillie et la mise bas. Aucune interaction significative entre le régime et le stade de gestation n'est à noter, bien que la rétention azotée ait tendance à augmenter en fin de gestation sous l'effet d'un apport énergétique accru.

TABLEAU 4

Influence du stade de gestation sur la rétention azotée $(\mathrm{g} / \mathrm{j})$

\begin{tabular}{|c|c|c|c|c|c|}
\hline Stade de gestation (j) & 38 à 41 & 59 à 62 & 80 à 83 & 101 à 104 & Effet du stade \\
\hline $\begin{array}{l}\text { Lot } 1 \text { (témoin) } \ldots \ldots \ldots \\
\text { Lot } 2(+ \text { rafles }) \ldots \ldots \\
\text { Lot } 3(+ \text { amidon }) \ldots\end{array}$ & $\begin{array}{l}14,2 \\
16,2 \\
15,6\end{array}$ & $\begin{array}{l}11,3 \\
14,1 \\
15,8\end{array}$ & $\begin{array}{l}10,8 \\
14,0 \\
17,9\end{array}$ & $\begin{array}{l}12,1 \\
15,6 \\
18,1\end{array}$ & $\begin{array}{l}\text { NS } \\
\text { NS } \\
P \leqslant 0,10\end{array}$ \\
\hline
\end{tabular}

\section{C. - Intensité de synthèse des lipides et composition en acides gras du lard dorsal des truies (tabl. 5 et 6 )}

L'intensité de synthèse des lipides, estimée in vitro sur un échantillon de lard prélevé par biopsie au niveau du cou des truies, tend à diminuer chez tous les animaux entre le début et la fin de la gestation (tabl. 5). Mais l'effet du stade n'est significatif que pour la production de $\mathrm{CO}_{2}$ marqué qui, dans tous les cas, évolue parallèlement à la synthèse des lipides.

\section{TABLEAU 5}

Résultats d'incubation de coupes de tissu adipeux dorsal des truies en présence de glucose uniformément marqué

(exprimés en nanomoles de substrat transformé en $\mathrm{CO}_{2}$, ou en lipides totaux pour roo $\mathrm{mg}$ de tissu et 2 heures d'incubation)

\begin{tabular}{c|c|c|c|c}
\hline & $\begin{array}{c}\text { Stade de } \\
\text { gestation }(\mathrm{j})\end{array}$ & $\begin{array}{c}1 \\
\text { Témoin }\end{array}$ & $\begin{array}{c}2 \\
+ \text { rafles }\end{array}$ & $\begin{array}{c}\text { Lots } \\
+ \text { amidon }\end{array}$ \\
\hline $\begin{array}{c}\text { Substrat transformé } \\
\text { en } \mathrm{CO}_{2}\end{array}$ & 26 & $30,4 \pm 9,7$ & $25,7 \pm 6,0$ & $69,5 \pm 9,0$ \\
\hline $\begin{array}{c}\text { Substrat transformé } \\
\text { en lipides totaux }\end{array}$ & 108 & $20,5 \pm 9,3$ & $15,4 \pm 5,9$ & $23,8 \pm 4,9$ \\
\hline
\end{tabular}

Chaque valeur moyenne est accompagnée de son écart-type ( 3 données par lot). 
On ne note aucune différence dans les taux d'incorporation de précurseur marqué dans les lipides ou le $\mathrm{CO}_{2}$ formé entre les lots I et 2. Par contre, dans le lot 3 , l'incorporation du glucose tend à être plus importante que dans les autres deux lots, tout au moins au $26^{\mathrm{e}}$ jour de gestation. Mais, étant donné leur variabilité élevée, ces valeurs ne sont pas statistiquement différentes.

La composition relative en acides gras du lard dorsal des truies (tabl. 6) ne montre pas de variations importantes. Le régime des animaux ne l'affecte pas, et seul le stade de gestation exerce une influence significative, le pourcentage d'acides palmitique et stéarique diminuant entre le $26^{\mathrm{e}}$ et $1 \mathrm{e}$ Io $8^{\mathrm{e}}$ jour après la saillie. Cette baisse est compensée par une augmentation de certains autres acides gras, oléique en particulier. D'une façon générale, on constate que la teneur des lipides en acides gras saturés tend à diminuer (de 35,7 à 33,2 p. I0o), alors que celle des acides insaturés totaux s'élève.

\section{TABLEAU 6}

Composition moyenne en acides gras du lard dorsal des truies (p. Iоo)

\begin{tabular}{|c|c|c|c|c|c|c|c|c|c|}
\hline Lot & $\begin{array}{l}\text { Stade de } \\
\text { gestation } \\
\text { (j) }\end{array}$ & $\mathrm{C}_{14}$ & $C_{16}$ & $C_{16: 1}$ & $\mathrm{C}_{18}$ & $C_{18: 1}$ & $C_{18: 2}$ & $C_{20}$ & $C_{18: 3}+C_{20: 1}$ \\
\hline$\stackrel{1}{\text { témoin }}$ & $\begin{array}{r}26 \\
108\end{array}$ & $\begin{array}{l}1,5 \\
1,4\end{array}$ & $\begin{array}{l}23,4 \\
21,8\end{array}$ & $\begin{array}{l}3,4 \\
3,5\end{array}$ & $\begin{array}{r}10,7 \\
9,2\end{array}$ & $\begin{array}{l}47,3 \\
50,3\end{array}$ & $\begin{array}{l}10,9 \\
11,1\end{array}$ & $\begin{array}{l}0,1 \\
0,5\end{array}$ & $\begin{array}{l}2,7 \\
2,3\end{array}$ \\
\hline $\begin{aligned} & 2 \\
+ & \text { rafles }\end{aligned}$ & $\begin{array}{r}26 \\
108\end{array}$ & $\begin{array}{l}1,7 \\
1,3\end{array}$ & $\begin{array}{l}23,6 \\
22,1\end{array}$ & $\begin{array}{l}3,1 \\
3,3\end{array}$ & $\begin{array}{r}10,0 \\
9,5\end{array}$ & $\begin{array}{l}48,2 \\
48,9\end{array}$ & $\begin{array}{l}10,7 \\
12,6\end{array}$ & $\begin{array}{l}0,2 \\
0,5\end{array}$ & $\begin{array}{l}2,4 \\
1,7\end{array}$ \\
\hline $\begin{array}{c}3 \\
+ \text { amidon }\end{array}$ & $\begin{array}{r}26 \\
108\end{array}$ & $\begin{array}{l}1,4 \\
1,4\end{array}$ & $\begin{array}{l}22,8 \\
22,6\end{array}$ & $\begin{array}{l}3,3 \\
3,4\end{array}$ & $\begin{array}{r}10,8 \\
8,9\end{array}$ & $\begin{array}{l}47,7 \\
50,7\end{array}$ & $\begin{array}{l}11,4 \\
11,3\end{array}$ & $\begin{array}{l}0,8 \\
0,5\end{array}$ & $\begin{array}{l}1,9 \\
1,3\end{array}$ \\
\hline \multicolumn{2}{|c|}{$\begin{array}{l}\text { Effet du stade } \\
\text { de gestation }\end{array}$} & NS & ** & NS & $* * *$ & NS & NS & NS & NS \\
\hline
\end{tabular}

\section{D. - Performances de reproduction (tabl. 7 et 8 )}

En raison du faible nombre de truies par lot, les résultats de reproduction, qui sont rapportés dans le tableau 7 , ne sont donnés qu'à titre indicatif. Bien entendu, ils ne montrent aucun effet significatif du régime maternel sur le nombre ou le poids des porcelets à la naissance. Remarquons toutefois que le gain de poids total de gestation est le plus élevé chez les animaux du lot 3 , soit $7 \mathrm{I} \mathrm{kg}$ contre 49 et $4 \mathrm{I}$ respectivement dans les lots I et 2 . Il en est de même pour le gain net, qui passe de 37 et $28 \mathrm{~kg}$ dans les lots I et 2 à $5^{8} \mathrm{~kg}$ dans le lot 3 .

La composition chimique des porcelets à la naissance (tabl. 8) n'est pas influencée par les régimes maternels de gestation étudiés. Que les résultats soient rapportés au poids frais ou au poids sec, aucune différence entre les teneurs en matière sèche, protéines, lipides, minéraux ou énergie brute des animaux issus des truies des 3 lots n'est mise en évidence. De la même façon, les quantités totales de chacun de ces 
constituants dans les portées à la mise bas sont comparables d'un lot à l'autre ; dans tous les cas, elles présentent une corrélation élevée avec le poids vif des porcelets, soit respectivement $0,87,0,97$ et $0,9 \mathrm{I}$ pour l'azote, les minéraux et l'énergie.

\section{TABLEAU 7}

Résultats de reproduction

\begin{tabular}{|c|c|c|c|c|}
\hline Lot & $\begin{array}{c}1 \\
\text { Témoin }\end{array}$ & $\begin{array}{c}2 \\
+\quad \text { rafles }\end{array}$ & $\begin{array}{c}3 \\
+ \text { amidon }\end{array}$ & $\begin{array}{l}\text { Effet du } \\
\text { régime }\end{array}$ \\
\hline Gain total de gestation $(\mathrm{kg}) \ldots$ & $49^{a}$ & $41^{a}$ & $71^{b}$ & $* *$ \\
\hline Gain net de gestation $(\mathrm{kg}) \ldots$. & $37^{a}$ & $28^{a}$ & $58^{b}$ & $* *$ \\
\hline Nombre de porcelets nés ..... & 8,7 & 11,0 & 8,0 & NS \\
\hline Nombre de porcelets nés vivants & 8,3 & 7,3 & 7,7 & NS \\
\hline $\begin{array}{l}\text { Poids de la portée à la naissance } \\
(\mathrm{kg}): \text { porcelets vivants } \ldots . .\end{array}$ & 9,55 & 8,02 & 9,57 & NS \\
\hline $\begin{array}{l}\text { Poids moyen des porcelets à la } \\
\text { naissance }(\mathrm{kg}) \ldots \ldots \ldots \ldots \ldots\end{array}$ & 1,17 & 1,08 & 1,28 & NS \\
\hline
\end{tabular}

TABLEAU 8

Composition chimique des porcelets à la naissance

(3 animaux par lot)

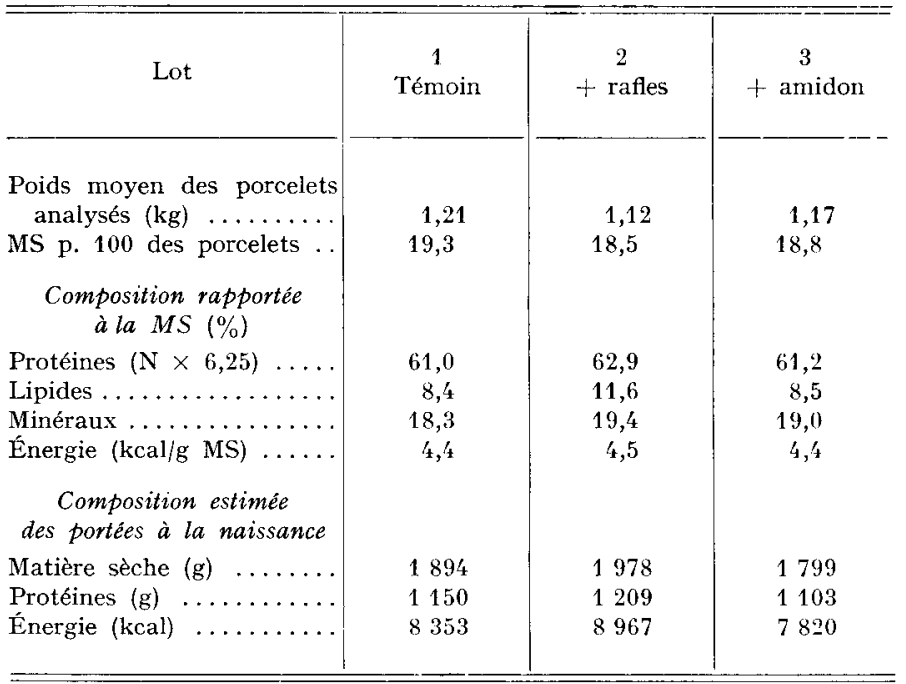

De cette expérience, on peut déduire que l'organisme des porcelets à la naissance contient en moyenne $\mathrm{I} 89 \mathrm{~g}$ de matière sèche, $\mathrm{I} 8,7$ d'azote, $\mathrm{I} 8, \mathrm{o} \mathrm{g}$ de lipides et $35,4 \mathrm{~g}$ de minéraux par $\mathrm{kg}$ de poids vif, leur teneur en énergie brute étant de $84 \mathrm{I} \mathrm{kcal} / \mathrm{kg}$, quel que soit le régime de leur mère au cours de la gestation. 


\section{DISCUSSION}

\section{A. - Nature de l'énergie et résultats de digestibilité}

Le présent travail montre que l'adjonction de rafles de maïs à la ration de base provoque une diminution de tous les coefficients d'utilisation digestive en comparaison avec l'aliment témoin. Ainsi que l'ont indiqué de nombreux travaux antérieurs, cet effet est dû à l'élévation du taux de cellulose brute du régime, qui passe de 2,3 à 7,0 p. IoO. L'effet dépressif sur la digestibilité de l'énetgie est de 6,3 points, soit un taux de diminution de I,34 p. Ioo pour I p. Ioo d'augmentation du taux de cellulose. Cette réduction est plus forte que celle que nous avions obtenue sur des porcs de $80 \mathrm{~kg}$ (HENRY et ÉTIENNE, I 969), soit 0,8 p. IOo avec une cellulose de bois purifiée ; elle est, par contre, moins élevée que dans d'autres travaux qui font état d'une diminution de 2 à $3 \mathrm{p}$. Ioo avec des régimes à base de céréales et d'issues de meunerie, riches en hémicelluloses (AxELsson, I955; NEHRING, I966; HENRY, I968) : en réalité, dans la présente expérience, les truies ingèrent des rafles de maîs contenant, outre la cellulose, de la lignine qui n'est pas digérée par le Porc (LaUrentowskA, I959), et une proportion réduite d'hémicelluloses. On peut invoquer plusieurs raisons à ces différences. Il ressort de certains travaux (HENRY, I97I) que les constituants membranaires totaux (cellulose + hémicelluloses + lignine) permettent une meilleure prévision de 1'utilisation digestive de l'énergie pour le Porc que la cellulose brute; cette dernière, en effet, ne tient pas compte de la fraction des hémicelluloses, dont l'utilisation digestive est relativement faible chez le Porc et comparable à celle de la cellulose. Ceci explique que l'effet dépressif, rapporté à la cellulose brute, ait été moins important que dans l'essai précédent. Par ailleurs, la participation de la cellulose à la couverture du besoin en énergie augmente avec l'âge du Porc, à cause de sa meilleure utilisation digestive, ainsi que l'ont montré CunNINGHAM et al. (I962), puis HENRY et ÉTIENNE (I969). Nos truies sont plus lourdes et plus âgées que les animaux en croissance d'AXELSSON (I955) et Henry (I968). Il est done logique que le bilan de 1'énergie digestible s'en trouve amélioré. La digestibilité propre de la cellulose brute est d'ailleurs relativement élevée, même lorsqu'elle est présente en proportion importante dans l'aliment. Lorsque son taux dans le régime diminue (lots I et 3), sa digestibilité apparente augmente, ainsi que l'ont trouvé FrIEND et al. (I962)., La variabilité élevée de ce critère n'est pas surprenante, comme l'ont constaté Zivkovic et Bowland (I970) et SAvic et al. (I972).

Ainsi qu'il a déjà été remarqué auparavant (HENRY et ÉTIENNE, I969), l'élévation du taux de cellulose du régime diminue la digestibilité des autres constituants de la ration, qu'il s'agisse de l'énergie ou des matières azotées. Cet effet peut être attribué à l'augmentation du volume de la ration (WoODMAN et EvaNs, I947), ou à l'accélération du transit qui est observée en particulier chez la Truie (RICHTER et Busch, I970), ce qui a pour conséquence une augmentation de l'excrétion endogène fécale.

Malgré cela, le bilan global montre que l'addition de $0,3 \mathrm{~kg}$ de rafles de maîs au régime de base élève de $5 \mathrm{I} 5 \mathrm{kcal}$ l'énergie digestible ingérée quotidiennement par les 
animaux, ce qui correspond à une augmentation du niveau énergétique de la ration de près de $9 \mathrm{p}$. Ioo. Il en résulte que, dans les conditions de cette expérience, les rafles de maïs apportent I $900 \mathrm{kcal}$ d'énergie digestible $/ \mathrm{kg}$ de matière sèche. Ce résultat est très supérieur aux estimations de BOENKER et al. (I969) qui trouvent que les rafles de maïs, introduites au taux de io p. Ioo dans l'aliment, n'ont aucune valeur énergétique pour le Porc en croissance-finition. Leurs travaux portaient en fait sur des animaux de 44 à $5^{8} \mathrm{~kg}$ de poids vif, tandis que nos truies pesaient entre I20 et $\mathrm{r} 60 \mathrm{~kg}$ ce qui pourrait expliquer, tout au moins partiellement, la différence constatée.

Dans le régime 3, supplémenté en amidon, les résultats de digestibilité des nutriments énergétiques (sauf la cellulose) sont supérieurs à ceux du régime témoin. Cet écart provient de la bonne utilisation de l'amidon de maïs, dont la matière sèche, la matière organique ou l'énergie brute sont complètement digérées (entre 1oo, 6 et Ior,6 p. Ioo d'après les calculs effectués par différence), en accord avec ScHiEmanN et al. (I96I). La valeur de l'amidon de maïs pour les truies peut être ainsi estimée à $4200 \mathrm{kcal}$ d'énergie digestible/kg de matière sèche.

\section{B. - Niveau des apports d'énergie et rétention azotée}

L'élévation des apports quotidiens d'énergie digestible à partir d'un niveau bas chez la 'Truie en gestation, provoque une amélioration de la rétention azotée, comme d'ailleurs des coefficients de rétention azotée. Ce résultat confirme l'existence d'une action d'épargne de l'énergie sur l'azote aussi bien chez les truies adultes (SALMON-LEgagneur, I963; FisLex, I967; Pike, I970) que chez les animaux en croissance (HENRY et DE WILDE, I973). Il apparaît, de plus, dans notre expérience que l'influence de l'augmentation des apports d'énergie sur le métabolisme azoté est d'autant plus marquée que le niveau initial est bas. Ainsi, entre les truies du lot I et celles du lot 2 , une ingestion d'énergie digestible supérieure de 8,8 p. Ioo améliore le coefficient de rétention azotée de $2 \mathrm{I}, 6 \mathrm{p}$. Ioo en valeur relative, tandis qu'entre les lots 2 et 3 , l'écart n'est plus que de I I p. Ioo pour une augmentation de consommation d'énergie de 44 p. Ioo.

Le niveau d'énergie digestible des truies qui reçoivent des rafles de maïs en plus de la ration de base diffère peu des normes du N. R. C. (I968) : $6600 \mathrm{kcal} /$ jour, l'apport azoté étant par ailleurs conforme aux recommandations ( $280 \mathrm{~g}$ de protéines brutes/ jour). Or, nos résultats montrent qu'une réduction faible des apports d'énergie digestible (lot I) par rapport aux normes provoque un abaissement de la rétention azotée des truies, alors que leur augmentation (lot 3) n'améliore qu'assez peu le bilan azoté. Il est possible de discuter la signification de ce critère dans le cas des truies gestantes, compte tenu de la faible sensibilité des résultats zootechniques à des modifications alimentaires importantes. Mais il ne faut pas oublier que ces truies auront à assurer, après la parturition, une production de lait élevée, dont la synthèse exige la participation des réserves maternelles, protéiques en particulier, édifiées pendant la gestation. D'autre part, la croissance des truies nullipares utilisées dans la présente expérience n'est pas terminée. Le bilan azoté de ces animaux revêt donc une signification particulière si l'on considère leurs performances de reproduction ultérieures. Sur la base de ce critère, il apparaît que les normes énergétiques actuelles (N. R. C., I968) des truies gestantes sont marginales, tout au moins lorsque celles-ci n'ont pas atteint leur poids adulte. 
A l'inverse de ce qui a été trouvé par plusieurs auteurs (Rombau'rs, r962; SALMON-LEGAGNEUR, I965 ; EISLEY et al., I966 a), nous n'obtenons pas d'amélioration de la rétention azotée des truies avec l'avancement de la gestation. A cela plusieurs explications peuvent être données. Nos bilans ne commencent qu'à partir de 40 jours après la saillie et sont déjà assez élevés. D'autre part, la rétention azotée varie de façon aléatoire, ainsi que l'ont montré des mesures effectuées toutes les semaines par SALMON-I,EGAGNEUR (I965). Einfin, les truies que nous avons utilisées sont encore en croissance, ce qui a pu interférer avec l'anabolisme gravidique. Remarquons cependant que la rétention azotée chez les truies du lot 3 augmente, bien que non significativement $(\mathrm{P} \leqslant 0, \mathrm{IO})$ entre 40 jours après l'accouplement et la mise bas. Comme cet effet n'existe pas chez les autres animaux, pour lesquels l'apport d'énergie digestible est plus limité, il apparaît qu'une telle restriction peut réduire non seulement le bilan azoté moyen des truies, mais aussi la "super rétention " azotée de fin de gestation (LENKEIT et al., I955). Ce résultat semble d'autant plus logique que la dépense d'énergie s'accroissait au cours de cette période, et nous voyons là un argument supplémentaire pour ne pas réduire les normes énergétiques actuellement en vigueur.

\section{C. - Niveau des apports d'énergie, lipogenèse et composition en acides gras $d u$ tissu adipeux}

L'intensité de la lipogenèse, mesurée in vitro sur des échantillons de lard dorsal des truies, diminue entre 26 et ro 8 jours de gestation, quel que soit le lot auquel appartiennent les animaux. Ces résultats sont à rapprocher de ceux de SALMonLEGAGNEUR (I963) et de ADAm et al. (I97I), qui observent que 1'épaisseur du lard dorsal, mesurée aux ultrasons, diminue en fin de gestation. SALMON-LEGAGNEur et JACQUOT (I96I), après dissection des carcasses de truies à différents stades, trouvent une diminution relative des tissus gras entre la saillie et la mise bas. Enfin, VERSTEGEN et al. (I97I) mettent en évidence, sur des truies placées en chambres respiratoires, une augmentation de la production d'extrachaleur au cours des 4 dernières semaines de gestation.

L'intensité de la lipogenèse est la plus importante à 26 jours de gestation pour les truies $\mathrm{du} 3^{\mathrm{e}}$ lot. Leurr régime, plus énergétique et à base d'amidon, favorise donc leur engraissement. Par contre, en fin de gestation, l'activité du tissu adipeux est la même pour tous les animaux. En d'autres termes, la synthèse de lipides de novo à partir d'un apport accru d'énergie, dans le lot 3, est alors abaissée au profit de la protéinogenèse. On peut invoquer l'augmentation des dépenses d'extrachaleur (VERSTEGEN et al., I97I), mais aussi l'élévation des besoins en énergie due à l'amélioration de la rétention azotée au fur et à mesure de l'avancement de la gestation, constatée chez les animaux du lot "amidon ".

Le régime de gestation ne semble pas influencer la composition en acides gras du tissu adipeux dorsal des truies. Par contre, cette composition évolue de la même façon pour les animaux des 3 lots entre le début et la fin de la gestation, la proportion d'acides gras insaturés augmentant au détriment des acides gras saturés. SALMON-LEGAGNEUR (I965) observe une diminution du pourcentage d'acide palmitique ou stéarique entre l'accouplement et la parturition. Mais, compte tenu des modifications qui portent sur la quantité de lipides stockés au cours de la gestation, 1'interpré- 
tation de ces variations est difficile. Elles peuvent aussi bien être dues à un stockage préférentiel des acides gras insaturés après la saillie qu'à une utilisation plus importante des acides gras saturés en fin de gestation. Il ne faut pas non plus négliger les interconversions de ces acides entre eux et aucune de ces 3 hypothèses ne peut être rejetée dans le cas présent.

\section{D. - Niveau énergétique et performances de reproduction}

Les conclusions qu'il est possible de tirer à partir des résultats d'élevage des truies sont conformes aux observations de la plupart des auteurs qui ont étudié 1'influence de l'apport d'énergie aux truies gestantes. Les animaux qui ingèrent le plus d'énergie digestible (lot 3) réalisent un gain total ou net de gestation supérieur aux autres, comme l'ont signalé EiLsLEy et al. (I968 b), VERMEDAHL et al. (Ig69) ou FroBish et al. (I973). On peut être surpris de ce que les truies du lot 2 aient des croissances inférieures à celles du lot témoin avant la parturition, bien que leur rétention azotée ait été plus importante. Peut-être ont elles stocké moins de lipides, ainsi que les mesures d'activité du tissu adipeux tendent à l'indiquer. Mais les résultats d'élevage sont à considérer avec précaution compte tenu du faible effectif d'animaux dans chaque lot. Aucune différence significative n'est mise en évidence en ce qui concerne les portées. Le nombre de porcelets nés ou nés vivants n'est pas influencé de façon statistique, ainsi que l'ont indiqué ELSLEY et al. (I968 b) et SRECKOvic et al. (I97I). La mortalité périnatale, plus élevée parmi les porcelets des truies du lot 2, est due en partie à leur poids moyen à la naissance assez faible, mais surtout au fait que les parturitions ont eu lieu dans une porcherie équipée d'un sol sur caillebotis. Le poids moyen des porcelets à la naissance tend à être supérieur lorsque leurs mères ont ingéré plus d'énergie digestible pendant la gestation (lot 3). Ce résultat est conforme aux observations de Frobish et al. (I973) et ELSLEY et al. (I969). La différence de poids entre les animaux issus des truies des lots I et 2 s'explique par la plus grande taille de portée dans le cas du lot 2.

L'absence d'effet du niveau et de l'origine des apports d'énergie aux truies gestantes sur la composition chimique des porcelets à la naissance a été signalée par plusieurs auteurs, dont ELSLEY et al. (Ig66 b) qui ont trouvé que pour des niveaux alimentaires de gestation compris entre $I, 6$ et $2,6 \mathrm{~kg} / \mathrm{j}$ le contenu azoté corporel des jeunes, de l'ordre de $18 \mathrm{~g} / \mathrm{kg}$, n'est pas modifié par le traitement antérieur de la truie. D'autres expériences, portant sur la quantité de protéines distribuées aux mères (Livingstone et al., I966 ; Pond et al., I968) aboutissent à des conclusions analogues, tout au moins en ce qui concerne la teneur en azote des porcelets à la mise bas, ou à l'abattage à $90 \mathrm{~kg}$.

En conclusion, les résultats de cette expérience confirment que les niveaux des apports d'énergie digestible considérés ont peu, ou pas, d'influence sur la reproduction des truies, tout au moins pendant un seul cycle. Pourtant, les mesures de rétention azotée indiquent qu'il est préférable de ne pas préconiser des normes énergétiques trop faibles pendant la gestation, notamment dans le cas des truies nullipares, encore en croissance. Nous rejoignons en cela les conclusions de FisLEY et al. (I968 a), selon lesquels la Truie peut, à la suite d'un rationnement trop sévère appliqué pendant plusieurs gestations, se trouver dans un état d'épuisement tel qu'elle serait incapable 
de mener à bien la lactation suivante. La présente étude montre en effet qu'à ưr apport d'énergie plus faible correspond non seulement une lipogenèse moins importante, mais aussi une rétention azotée réduite, ce qui diminue d'autant les réserves tissulaires maternelles utilisables pour la production laitière ultérieure.

Reçu pour publication en mai 1973.

\title{
SUMMARY
}

\author{
APPARENT DIGESTIBILITY AND METABOLIC UTILIZATION \\ OF NUTRIENTS AND REPRODUCTIVE PERFORMANCES \\ IN PREGNANT GILTS AS AFFECTED BY THE ENERGY SUPPLY
}

The present experiment was carried ont on 9 Large White gilts, mated at a live weight of about $\mathrm{I} 20 \mathrm{~kg}$, with the aim of studying the effect of the daily energy supplyy level during pregnancy on the apparent digestibility of nutrients, mitrogen retention and lipid deposition in the gilt, as well as on the chemical composition of the piglets at birth.

Three groups of 3 gilts each were compared. They received respectively a control dliet (group I) composed of maize and soybean oil-meal (I.8 kg/day), the same diet added with $0.3 \mathrm{~kg}$. maize cobs. (group 2) or with $0.9 \mathrm{~kg}$ maize starch (group 3). During pregnancy each animal was subjected to 4 periods of feces and urine collection with a view to determining the digestibility coefficients and gaitrogen retention. At the same time, the intensity of the lipogenesis was studied in vitro, on adipóse tissue slices from samples obtained by biopsy in the cervical area, by measuring the incorporation rate of a labelled precursor (glucose $-\mathrm{U}^{-14} \mathrm{C}$ ) into the synthetized lipids and the carbon. dioxide produced. The fatty acid composition of the adipose tissue was also studied. The trial was completed by observations pertaining to farrowing performances (number and weight of. the piglets) and total chemical composition of the piglets (one animal per litter).

The results obtained show that introduction of maize cobs into the diet, inducing rise of its: crude fiber content from 2.3 to $7 \mathrm{p}$. Ioo, led to decrease of all digestibility coefficients measured' (tabl. 2); from 86.5 to 80.2 p. Ioo in the case of energy for instance. The content of digestible energy in the raw materials added per kg dry matter was I 900 kcal for maize cobs, $4200 \mathrm{kcal}$ for maize starch. Although the nitrogen intake was the same for all gilts, the daily nitrogen. retention increased when raising the digestible energy intake level (tabl. 3), i.e. I2, I 5 and $17 \mathrm{~g}$ : respectively for groups I, 2 and 3, corresponding to 5.9, 6.4 and 9.2 Mcal digestible energy/lay. In addition, increase of the nitrogen retention during pregnancy only occurred in the animals: of group 3 (from I 5.6 to I8. I g nitrogen/day) (tabl. 4).

These results indicate that an excessive restriction of digestible energy in the gilts reduced: the body reserves of protein built up during pregnancy. Furthermore, synthesis of maternal lipids, measured in vitro (tabl. 5) was reduced during early pregnancy.

The energy supply level during pregnancy did not change either the litter size at birth. (tabl. 7) or the chemical composition of the piglets (tabl. 8). On the other hand, there was a tendency. for their mean weight and net gain to increase during pregnancy at the highest energy level. (group 3): the date recorded were $1.28 \mathrm{~kg}$ and $5^{8} \mathrm{~kg}$ respectively for group 3 versus I.I 7 and. $37 \mathrm{~kg}$ for group $\mathrm{I}$ and $\mathrm{I} .08$ and $28 \mathrm{~kg}$ for group 2 .

The present study shows that an exagerated restriction of digestible energy has an unfavourable effect on nitrogen retention in pregnant gilts and that the current energy standards. (N. R. C., 1968) are marginal for animals which have not yet completed their growth.

\section{RÉFÉRENCES BIBLIOGRAPHIQUES}

Adam J. L., Shearer I. J., Hargreaves K., r971. The effects of type and level of feeding of sows: during pregnancy. I. Sow performance. New Zeal. J. Agric. Res., 14, 646-660.

Axelsson J., r 955. Der Einfluss des Rohfaser Gehaltes des Futters auf das Wachstum von Jungschweinen. Arch. Tierernähr., 5, I-I6. 
Baker D. E., Becker D. E., Norton H. W., Sasse C. E., Jensen A. H., Harmon B. G., 1969. Reproductive performance and progeny development in swine as influenced by feed intake during pregnancy. J. Nutr., 97, 489-495.

Bligh E. G., Dyer W. J., I959. A rapid method of total lipid extraction and purification. Can. J. Biochem. Physiol., 37, 9II-9I7.

Boenker D. E., Tribble L. F., Pfander W. H., r969. Energy and nitrogen evaluation of swine diets containing added fat or corn cobs. J. Anim. Sci., 28, 615-6I9.

Cunningham H. M., Friend D. W., Nicholson J. W. G., ig62. The effect of age, body weight, feed intake and adaptability of pigs on the digestibility, and nutritive value of cellulose. Can. J.Anim. Sci., 42, 167-I75.

ElsLey F. W. H., I967. Nutrition of the pregnant sow. Proceedings of a symposium on the nutrition of sores, Nottingham, pp. zo-26; Mary Bannerman ed., Rowett Research Institute.

Elsley F. W. H., Anderson D. M., McDonald I., MacPherson R. M., Smart R., ig66a. A comparison of the live-weight changes, nitrogen retention and carcass composition of pregnant and nonpregnant gilts. Anim. Prod., 8, 39I-400.

Elsley F. W. H., McDonald I., MacPherson R. M., i 966 . The effect of feed intake and protein concentration during pregnancy on the nitrogen content of newly born pigs. Anim. Prod., 8, 353 (Abstr.).

Elsley F. W. H., MacPherson R. M., Lodge G. A., ig68a. The effects of level of feeding of sows during pregnancy. III. Body composition. Anim. Prod., 10, 149-1 56.

Elsley F. W. H., MacPherson R. M., McDonald I., Ig68 $b$. The influence of dietary energy in pregnancy and lactation upon sow productivity. J. Agric. Sci., 71, 21 5-222.

Elsley F. W. H., Bannerman M., Bathurst E. V. J., Bracewell A. G., Cunningham J. M. M., Dodsworth T. L., Dodds P. A., Forbes T. J., LAird R., I969. The effect of level of feed intake in pregnancy and in lactation upon the productivity of sows. Anim. Prod., 11, 225-24I.

Evans R. E., 1929. Protein and mineral metabolism of pregnant sows on a normal or high calcium diet. J. A gric. Sci., 19, $752-798$.

Friend D. W., Cunningham H. M., Nicholson J. W. G., ig62. The production of organic acids in the pig. I. The effect of diet on the proportions of volatile fatty acids in pig feces. Can.J.Anim. Sci., 42, 55-62.

Frobish L. T., Stefle N. C., Davey R. J., I973. Long term effect of energy intake on reproductive performance of swine. J. Anim. Sci, 36, 293-297.

Henry Y., ig68. Influence des variations du taux de cellulose ou d'un diluant inerte du régime sur les performances de croissance et l'utilisation digestive des nutriments chez le Porc. 2nd World Conference on Animal Production, Short communications, Univ. Maryland, College Park, U. S. A.

Henry Y., Ig7r. Essai de prévision de la valeur en énergie digestible des aliments pour le Porc, à partir de leurs teneurs en constituants membranaires. Journées de la Rech. Porcine en France, I. N. R. A., Paris, I. T. P. Ed., 57-64.

Henry Y., Étienne M., Ig69. Effets nutritionnels de l'incorporation de cellulose purifiée dans le régime du Porc en croissance-finition. I. Influence sur l'utilisation digestive des nutriments. $A n n$. Zootech, , 18, 337-357.

HenRY Y, DE WIIDE R., I973. Incorporation de proportions variables de matières grasses (huile d'arachide) dans le régime du Porc en croissance-finition, en relation avec le taux de matières azotées. I. Influence sur I'utilisation digestive des constituants énergétiques et azotés, et la rétention azotée. Ann. Zootech. 22, I67-184.

LaURentowska C., I959. Capacité du porc à digérer la cellulose brute, la lignine, la cellulose et les pentosanes (en polonais). Rocz. Nank. Rol., $74 \mathrm{~B}, 567-578$.

Lenkeit W., Gütte J. O., Warnecke W., Kirchhoff W., 1955. Langfristige Untersuchen zum aüsseren und inneren Stoffwechsel des graviden und laktierenden Schweines. 3 Mitteilung : die Beziehung der N-Retention während das Gravidität zum N Umsatz nach der Geburt bei hoher Milchleistung und bei verhinderter Lacktation. Zeitschr. Tierernähr. Futtermittelk., 10, 351-364.

Livingstone R. M., MacPherson R. M., Elshey F. W. H., Lucas I. A. M., Lodge G. A., 1966. A note on the effect of protein concentration in the diets of pregnant sows on the performance and carcass quality of their progeny. Anim. Prod., 8, 337-339.

Lodge G. A., McDonald I., MacPherson R. M., I96I. Weight changes in sows during pregnancy and lactation. Anim. Prod., 3, 269-275.

Lodge G. A., Elsley F. W. H., MacPherson R. M., ig66. The effects of level of feeding of sows during pregnancy. I. Reproductive performance. Anim. Prod., 8, 29-38.

Miller G. M., Becker D. E., Jensen A. H., Harmon B. G., Norton H. W., ig69. Effect of protein intake on nitrogen retention by swine during late pregnancy. J. Anim. Sci., 28, 204-207.

Mitchell H. H., Carroll W. E., Hamilton T. S., Hunt G. E., I93I. Food requirements of pregnancy in swine. Bull. Ill. A gric. Exp. Stn. 375, 465-504.

Moustgaard J., 1962. Fœtal nutrition in the pig. In Nutrition of pigs and poultry. Butterworths London, 1 89-205.

Nrhring K., I 966. Rohfaser oder Rohcellulose. Tin Beitrag zur Entwicklung der weender Futtermittelanalyse. Arch. Tierernähr., 16, 77-102. 
N. R. C., I964. Nutrient Requirements for Domestic Animals, $n^{\circ}$ 2. Nutrient Requirements for swine. National Research Council, Washington, D. C., $\mathrm{n}^{0} \mathrm{I} 192$.

N. R. C., I968. Nutrient Requirements for Domestic Animals, no 2. Nutrient Requirements for swine. National Research Council, Washington, D. C. n' ${ }^{\circ} 599$.

O'HeA E. K., Leveille G. A., 1968. Lipogenesis in isolated adipose tissue of the domestic chick (Gallus domesticus). Comp. Biochem. Physiol., 26, 1081-1089.

PIKE I. H., r970. The effect on nitrogen utilization in the sow of variation in dietary protein concentration and pattern of feeding in pregnancy. J. Agric. Sci., 74. 209-2 I5

Pond W. G., Dunn J. A., Wellington G. H., Stovffer J. R., Van Vleck L. 1), ig68. Weight gain and carcass measurements of pigs from gilts fed adequate vs. protein-free diet during gestation. $J$. Anim. Sci., 2\%, 1583-1586.

Richter K., Busch B., I970. Prüfung von Rationen mit unterschiedlichen Energie-, Rohfaser- und Wassergehalt an graviden Sauen hinsichtlich Verdaulichkeit und Durchgangszeiten. Arch. Tievernähr. 20, $679-686$.

Rippel R. H., Harmon B. G., Jensen A. H., Norton H. W., Becher J). E., I965. Response of the gravid gilt to levels of protein as determined by nitrogen balance. J. Anim. Sci, 24, 209-2I5.

Rombavts P., i 962 . Évolution de l'anabolisme gravidique chez la Truie en fonction de l'âge de l'animal. Ann. Zootech., 11, 39-51.

Salmon-Legagneur li., ig63. Influence relative de 'azote et de l'énergie de la ration sur le comportement nutritionnel de la Truie gestante : anabolisme gravidique, protéinogenèse, lipogenèse et évolution du contenu utérin. Arch. Sci. Physiol., 17, 233-245.

Salmon-Legagneur E., I965. Quelques aspects des relations nutritionnelles entre la gestation et la lactation chez la Truie. Ann. Zootech., 14, no hors série I. I35 pp.

Salmon-LegagneUR 1i., ig69. Fitude de l'utilisation de différentes graisses par la Truie en gestation. Journées de la Rech. Porcine en France, Paris, I, N. R, A, I. T. P. I. I., II9-I24.

Salmon-Legagneur E., Jacquot R., i96I. Modifications corporelles entrainées par l'anabolisme gravidique chez la Truie. C.R. Acad.Sci., 253, 544-546.

Savic S., Kosanovic M., Sljivovacki K., Ivos K., I97r. The effect of energy and protein relation upon the digestibility and nitrogen retention of pregnant sows. Xe Congrès intern. de Zootechnie, Versailles.

Schiemand R., Hoffmann L., Nehring K., I96r. Die Verwertung reiner Nährstoffe. 2-Mitteilung : Versuche mit Schweinen. Arch. Tierernähr., 11, 265-283.

Sreckovic A., Bokorov T., Zrvkovic S., Nikolic M., I97i. The effect of different energy levels in the ration on productive and reproductive abilities of gilts and sows. Xe Congrès intern. de Zootechnie, Versailies.

Vermedahi, L. D., MEAde R. J., HaNke H. E., Rust J. W., ig69. Effects of energy intake of the dam on reproductive performance, development of offspring and carcass characteristics. J. Anim.Sci., 28, 465-472.

Verstegen M. W. A., Van Es A. J. H., Nijkamp N. J., rg7r. Some aspects of energy metabolism of the sow during pregnancy. Anim. Prod., 13, 677-684.

Woodman H. E., Evans R. E., I947. The nutritive value of fodder cellulose from wheat straw. II. The utilization of cellulose by growing and fattening pigs. $J$. A gric. Sci., 37, 2 I I-22I.

Zivkovic S., Bowland J. P., I970. Influence of substituting higher fiber ingredients for corn on the digestibility of diets and performance of sows and litters. Can. J. Anim. Sci., 50, I77-184. 